Acta Crystallographica Section B

Structural

Science

ISSN 0108-7681

Editor: Carolyn P. Brock

\title{
Isonicotinic acid
}

\section{F. Takusagawa and A. Shimada}

This electronic document was scanned from an archival copy of material deposited to accompany a paper published in an IUCr journal. In many cases the only accessible copy was a microfilm of a poor-quality original. 
.

\begin{tabular}{|c|c|c|c|c|c|c|c|c|c|c|c|c|c|c|}
\hline H & K & $\mathrm{E}$ & Fo & $F C$ & $t$ & $k$ & $L$ & $\mathrm{FO}$ & $\mathrm{FC}$ & $\mathrm{HI}$ & $k$ & $\mathrm{~L}$ & 75 & FC \\
\hline 2 & 0 & 0 & 688 & -12 & -3 & 4 & 0 & 23 & 22 & 6 & -8 & 1 & 4 & 3 \\
\hline 3 & 0 & 0 & 21 & 19 & -2 & 4 & 0 & 6 & 6 & -2 & -7 & 1 & 22 & 23 \\
\hline 4 & 0 & 0 & 170 & 179 & -1 & 4 & 0 & 41 & $\Delta 1$ & -1 & -7 & 1 & 16 & 17 \\
\hline 5 & 0 & 0 & 6 & 5 & 0 & 4 & 0 & 24 & 22 & 0 & -7 & 1 & 46 & 49 \\
\hline 6 & 0 & 0 & 32 & 31 & 1 & 4 & 0 & 52 & 55 & 1 & -7 & 1 & 15 & 21 \\
\hline 7 & 0 & 0 & 3 & 3 & 2 & 4 & 0 & 37 & 34 & 2 & -7 & 1 & 46 & $\$ 8$ \\
\hline 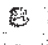 & 0 & 0 & 3 & 3 & 3 & 4 & 0 & 35 & 34 & 3 & -7 & 1 & $<3$ & 25 \\
\hline 8 & 1 & 0 & 5 & 4 & 4 & 4 & 0 & 29 & 26 & 4 & -7 & 1 & 10 & 11 \\
\hline 7 & 1 & 0 & 21 & 20 & 5 & 4 & 0 & 13 & 12 & 5 & -7 & 1 & 16 & 16 \\
\hline-8 & 1 & $\circ$ & 9 & 7 & $-e$ & 5 & 0 & 24 & 22 & 6 & -7 & 1 & 14 & 15 \\
\hline 5 & 1 & 0 & 19 & 18 & -7 & 5 & 0 & 93 & $\mathrm{GA}$ & 7 & -7 & 1 & 3 & 3 \\
\hline-19 & 1 & 0 & 5 & 6 & -6 & 5 & 0 & $\triangle 2$ & 42 & -4 & -6 & 1 & 7 & 6 \\
\hline-3 & 1 & 0 & 16 & 17 & -5 & 5 & 0 & 145 & 145 & -3 & -6 & 1 & 5 & 4 \\
\hline 12 & 1 & 0 & 14 & 13 & -4 & 5 & 0 & 46 & $\triangle 8$ & -2 & -6 & 1 & 16 & 15 \\
\hline-2 & 1 & 0 & 29 & 31 & -3 & 5 & 0 & 112 & 118 & -1 & -6 & $\rrbracket$ & 7 & 7 \\
\hline 0 & 2 & 0 & 66 & 63 & -2 & 5 & 0 & 33 & 38 & 0 & -6 & I & 43 & 43 \\
\hline 2 & 1 & 0 & ?o & $e s$ & -1 & 5 & 0 & 13 & 13 & 1 & -6 & 2 & 0 & 1 \\
\hline 2 & 1 & 0 & 80 & 88 & 0 & 5 & 0 & 25 & 26 & 2 & -6 & 1 & 36 & 36 \\
\hline 3 & 1 & 0 & 58 & 60 & 1 & 5 & 0 & 34 & 32 & 3 & -6 & 1 & 7 & 5 \\
\hline 3 & 1 & 0 & 41 & 40 & 2 & 5 & 0 & 12 & 12 & 4 & -6 & 1 & 10 & 9 \\
\hline 5 & 1 & 0 & 25 & 26 & 3 & 5 & 0 & 19 & 16 & 5 & -6 & 1 & 15 & 15 \\
\hline 5 & 1 & 0 & 13 & 12 & 4 & 5 & 0 & 2 & 2 & 6 & -6 & 1 & 0 & 1 \\
\hline 7 & 1 & 0 & 5 & 4 & $-\mathbf{z}$ & 6 & 0 & 4 & 5 & 7 & -6 & 1 & 2 & 4 \\
\hline-9 & 2 & 0 & 19 & $17:$ & -7 & 6 & 0 & 5 & 5 & 8 & -6 & 1 & 0 & 2 \\
\hline-7 & 2 & 0 & 15 & 13. & -6 & 6 & 0 & 0 & 0 & -5 & -5 & 1 & 6 & 5 \\
\hline-16 & 2 & 0 & 46 & 40 & -5 & 6 & 0 & 0 & 0 & -4 & -5 & 8 & 16 & 15 \\
\hline-5 & 2 & 0 & 28 & 28 & -4 & 6 & 0 & 13 & 13 & -3 & -5 & 1 & 11 & 10 \\
\hline - & 2 & 0 & 42 & 330 & -3 & 6 & 0 & 12 & 9 & -2 & -5 & 1 & 20 & 19 \\
\hline-3 & 2 & 0 & 40 & 44 & -2 & 6 & 0 & 12 & 12 & -1 & -5 & I & 26 & 25 \\
\hline-3 & 2 & 0 & 95 & 90. & -1 & 6 & 0 & 0 & 1 & 0 & -5 & 1 & 79 & 89 \\
\hline-1 & 2 & 0 & 83 & 85 & .0 & 6 & 0 & 10. & 9 & 1 & -5 & 1 & 33 & 32 \\
\hline 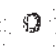 & 2 & 0 & 199 & 207 & 1 & 6 & 0 & 10 & 8 & 2 & -5 & 1 & E6 & ६.4 \\
\hline 1 & 2 & 0 & $\$ 0$ & 101 & 2 & 6 & 0 & 14 & 13 & 3 & -5 & 1 & 24 & 23 \\
\hline 2 & 2 & 0 & 107 & 113 & $z^{6}$ & 6 & 0 & 5 & 5 & 4 & -5 & $\mathbb{1}$ & 10 & 9 \\
\hline 3 & 2 & 0 & 53 & 54 & -71 & 7 & 0 & 21 & 22 & 5 & -5 & 1 & 22. & 22 \\
\hline 雪 & 2 & 0 & 21 & 18 & -6 & 7 & 0 & 0 & 2 & 6 & -5 & 1 & 41 & 42 \\
\hline 5 & 2 & 0 & 21 & 13 & -5 & 7 & 0 & $\$ 0$ & 44 & 7 & -5 & 1 & 22 & 21 \\
\hline 5 & 2 & 0 & 7 & 6 & -4 & 7 & 0 & 7 & 6 & 8 & $-5^{2}$ & 1 & 34 & 37 \\
\hline 7 & 2 & 0 & 6 & 6 & -3 & 7 & 0 & 32 & 30 & -6 & -4 & $\lambda$ & $c$ & 0 \\
\hline-8 & 3 & 0 & 10 & 9. & -2 & 7 & 0 & 15 & 15 & -5 & -4 & 1 & 15 & 12 \\
\hline-7 & 3 & 0 & 0 & 4 & -1 & 7 & 0 & 14 & 5 & -4 & -4 & $\mathbb{1}$ & 12 & 12 \\
\hline-5 & 3 & 0 & 27 & 24 & 0 & 7 & 0 & 11 & 13 & -3 & $\rightarrow$ & 1 & 30 & 29 \\
\hline-5 & 3 & 0 & 3 & 4 & 1 & 7 & 0 & 19 & 19 & -2 & -4 & $\$$ & 54 & 60 \\
\hline-1 & 3 & 0 & 30 & 31 & 2 & 7 & 0 & 8 & 5 & -2 & -4 & I & 40 & 40 \\
\hline-3 & 3 & 0 & 105 & 108 & -6 & $\varepsilon$ & 0 & 62 & 57 & 0 & -4 & 1 & 112 & 125 \\
\hline-2 & 3 & 0 & 35 & 31 & -5 & e & 0 & 5 & 4 & 1 & -4 & 1 & 35 & 35 \\
\hline-1 & 3 & 0 & 270 & 279 & -4 & a & 0 & 67 & 64 & 2 & $-4:$ & 1 & 101 & 104 \\
\hline 0 & 3 & 0 & 46 & $\$ 6$ & -3 & 8 & 0 & 0 & 1 & 3 & -4 & $i$ & 28 & 27 \\
\hline 1 & 3 & 0 & 228 & 232, & -2 & 8 & 0 & 40 & 41 & 4 & -4 & 1 & 43 & 42 \\
\hline 2 & 3 & 0 & 35 & 37 & -1 & $\varepsilon$ & 0 & 0 & 0 & 5 & $一$ & 1 & 17 & 16 \\
\hline 3 & 3 & 0 & $E 7$ & 89 & 3 & -9 & $1-$ & 9 & 7 & $\sigma$ & $-\$$ & 1 & 19 & 18 \\
\hline a & 3 & 0 & 14 & 13 & 4 & -5 & 1 & 5 & 5 & 7 & -4 & 1 & 0 & 2 \\
\hline 5 & 3 & 0 & 24 & 24 & -1 & $-e$ & 1 & 4 & 3 & 8 & -4 & 1 & 8 & 9 \\
\hline 5 & 3 & 0 & 0 & 1 & 0. & -8 & 1 & 18 & 18 & -7 & -3 & 1 & 7 & 7 \\
\hline-8 & 8 & 0 & 29 & 28 & 1 & -8 & 1 & 5 & 5 & -6 & -3 & 2 & 7 & 6 \\
\hline-7 & 4 & 0 & 28 & 21 & 2 & -8 & 1 & 18 & 13 & -5 & -3 & 8 & 13 & 21 \\
\hline-6 & 4 & 0 & 30 & 25 & 3 & -8 & 1 & 23 & 22 & -4 & -3 & 2 & 21 & 20 \\
\hline-5 & 4 & 0 & 25 & 26 & 4 & $-e$ & 1 & 8 & 4 & -3 & -3 & 2 & 2 & 3 \\
\hline-6 & $\Leftrightarrow$ & 0 & 5 & 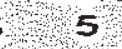 & 5 & -8 & 13 & 35 & 40 & -2 & -3 & 1 & 102 & 104 \\
\hline
\end{tabular}







H K R L FO FC U H K L FC FC

$6-7$ 2 21 20 $-6 \quad-2$ 2 42 \&

$7-7$ \& 606

$-6,217,87$

$\begin{array}{llll}-3 & -6 & 2 & 15 \\ -2 & -6 & 2 & 10\end{array}$

$-1 \quad-6 \quad 2 \quad 17,16$

- $-6 \mathrm{l}^{2} 16,15$

$2-6,2$ 18 15

$3=-6 \quad 2 \quad 50 \quad 5$

$\because-6 \quad 2 \quad 0 \quad 0$

$\therefore \begin{array}{rlll}-5 & -6 & 2 & 23 \\ -6 & 2 & 14\end{array}$

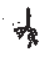

$7-6$

$-5 \quad-5$

$\begin{array}{ll}- & -5 \\ -3 & -5\end{array}$

$\begin{array}{ll}-2^{-} & -5 \\ -1 & -5\end{array}$

-

$$
\text { (1) }
$$

$$
3
$$

$\begin{array}{llllll}3 & -5 & 2 & 76 & 36\end{array}$

- $-5 \quad 2 \quad 920$

$\begin{array}{llllll}-1 \quad-2 & 2 \quad 100 \quad 107\end{array}$

$\begin{array}{cllll}-2 & 2 & 83 & 87\end{array}$

$\begin{array}{llll}6 & -2 & 2 & 3\end{array}$

$17 \mathrm{a}^{\circ}-7-1 \mathrm{a}^{2} 00$

5500

$\begin{array}{llllll}-6 & -1 & 2 & 13 & 13\end{array}$

$2-1 \quad 26671$

a $-2-1$

$\begin{array}{rr}-1 & -1 \\ 0 & -1\end{array}$ $\begin{array}{llll}-5 & -2 & 2 & 17 \\ 16\end{array}$

$-4-2 \quad 113107$

$1-3-2 \quad 2 \quad 34 \quad 37$

$-\bar{x}-2 \quad 2177$ 196

$3-2 \quad 2 \quad 122124$

• 2 -2 2 $252 \quad 58$

$3 \quad 3-2 \quad 20258$

$\therefore-\pi \quad 248$ \&E

$\begin{array}{rrrrrr}5 & -2 & 2 & 17 & 16\end{array}$

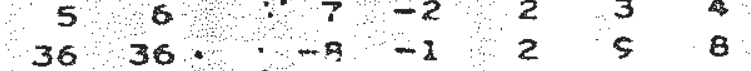

$.0-5+1222$

$1 .-3 \quad \begin{array}{rrrr}-1 & 2 & 10 & 0\end{array}$

276

2000

$\begin{array}{rrrrrrrrrr}6 & -5 & 2 & 0 & 2 & 3 & -1 & 2 & 8 & 8 \\ 7 & -5 & 2 & 0 & 0 & 4 & -1 & 2 & 35 & 33 \\ -6 & -4 & 2 & 6 & 6 & 5 & -1 & 2 & 19 & 17\end{array}$

$\begin{array}{lllllllllllll}-5 & -6 & 2 & 2 & 16 & 0 & -1 & 2 & 16 & 15\end{array}$

$-4 \quad 2 \quad 1928$

$\begin{array}{lllll}-3 & -4 & 2 & 27 & 26\end{array}$

$\begin{array}{lllll}-2 & -3 & 2 & 34 & 3\end{array}$

$-1 \quad 2 \quad 2 \quad 13 \quad 15$

$0-2527$

$1 \rightarrow 4 \quad 2 \quad 91 \quad 55$

$.2-121716$

$\begin{array}{lllll}3-4 & 2 & 88 & 8 \\ 4 & -3 & 2 & 22 & 2\end{array}$

$5-42240 \quad 43$

$6-4218$

$7-4 \quad 21612$

$8-22 \Rightarrow 6$

$\begin{array}{llll}-7 & -3 & 2 & 5 \\ -6 & 5\end{array}$

$\begin{array}{llllr}-6 & -3 & 2 & 26 & 26 \\ -5 & -3 & 2 & 5\end{array}$

$-\quad-325658$

$\begin{array}{llll}-3 & -3 & 2 & \end{array}$

$-2-3 \quad 258 \quad 54$

$\begin{array}{lllll}-1 & -3 & 2 & 22 & 23\end{array}$

$0 \quad-324.6$

$-32132128$

$2-3 \quad 21918$

$3-3 \quad 2.42145$

$217 \quad 18$

21516

210298

24643

2177179

2 1919

$236 \quad 37$

288

2100120

21918

259103

$216 \quad 16$

24753

288

$\begin{array}{lll}2 & 8 & 8\end{array}$

$230 \quad 28$

$236 \quad 35$

25654

2106112

26871

$\begin{array}{rrrrr}-1 & 1 & 2 & 75 & 82 \\ 0 & 1 & 2 & 55 & 57\end{array}$

$\begin{array}{rrrrr}-1 & 1 & 2 & 75 & 82 \\ 0 & 1 & 2 & 55 & 57\end{array}$

$\begin{array}{cccc}5 & -3 & 2 & 82 \\ 6 & -3 & 2 & 16\end{array}$

$7 \quad-3$ 2 2528

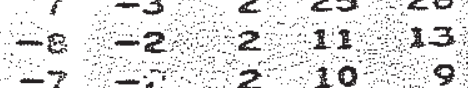

2. $20 \quad 11$

25453

$\begin{array}{rrr}2 & 10 & 9 \\ 2 & 6 & 5\end{array}$

202

2146143

H K L L FO FC

5112265

$\begin{array}{ccccc}6 & 1 & 2 & 4\end{array}$

$-8 / 2,2 \quad 5 \quad 5$

$\begin{array}{lrrrr}-7 & 2 & 2 & 9 & 8 \\ -6 & 2 & 2 & 31 & 32\end{array}$

$\begin{array}{lllll}-5 & 2 & 2 & 22 & 22\end{array}$

$-4 \quad 2 \quad 2119123$

$\begin{array}{llllll}-3 & 2 & 2 & 57 & 58\end{array}$

$\begin{array}{llllll}-2 & 2 & 2 & 155 & 199\end{array}$

$\begin{array}{lllll}-1 & 2 & 2 & 4 & 4\end{array}$

$\begin{array}{llllll}0 & 2 & 2 & 127 & 126\end{array}$

$\begin{array}{lllll}1 & 2 & 2 & 18 & 17\end{array}$

$2 \quad 2 \quad 240 \quad 38$

3221010

$4 \quad 2 \quad 2 \quad 97$

5:2 $24 \quad 5$

$\begin{array}{lllll}-9 & 3 & 2 & 16 & 16\end{array}$

$\begin{array}{llll}-8 & 3 & 2 & 4\end{array}$

$\begin{array}{lllll}-7 & 3 & 2 & 17 & 15\end{array}$

$\begin{array}{lllll}-6 & 3 & 2 & 28 & 29\end{array}$

$\begin{array}{lllll}-5 & 3 & 2 & 17 & 16\end{array}$

$\begin{array}{lllll}-4 & 3 & 2 & 70 & 72\end{array}$

$\begin{array}{lllll}-3 & 3 & 2 & 64 & 72\end{array}$

$\begin{array}{lllll}-2 & 3 & 2 & 68 & 76\end{array}$

$\begin{array}{lllll}-1 & 3 & 2 & 67\end{array}$

$\begin{array}{lllll}0 & 3 & 37 & 36\end{array}$

1 321011

$2 \quad 3 \quad 2 \quad 1414$

$\begin{array}{lllll}3 & 3 & 2 & 13 & 14\end{array}$

43267

$\begin{array}{llll}-9 & 4 & 0 & 2 \\ -9 & 4 & 15 & \end{array}$

$\begin{array}{llll}-8 & 2 & 15 & 15 \\ -7 & 2 & 12 & 12\end{array}$

$\begin{array}{lllll}-7 & 4 & 2 & 12 & 12 \\ -6 & 4 & 2 & 1 & 41\end{array}$

-5 \& 5152

$\begin{array}{llll}-4 & 4 & 52 & 53\end{array}$

$\begin{array}{llll}-3 & 2 & 78 & 77\end{array}$

-2 \& 2545

-142 a 53

0 2 3434

$1 \quad 2 \quad 25 \quad 23$

2 2 1618

3421010

$\begin{array}{lllll}-8 & 5 & 2 & 0 & 1\end{array}$

$\begin{array}{lllll}-7 & 5 & 2 & 3 & 3\end{array}$

$\begin{array}{lllll}-6 & 5 & 2 & 8 & 7\end{array}$

$\begin{array}{lllll}-5 & 5 & 2 & 54 & 62\end{array}$

$\begin{array}{lllll}-4 & 5 & 2 & 17 & 18\end{array}$

$-3 \quad 5 \quad 2 \$ 35136$

$\begin{array}{lllll}-2 & 5 & 2 & 16 & 16\end{array}$

$\begin{array}{lllll}-1 & 5 & 2 & 137 & 136\end{array}$

- 5 2 3 a

1527579

$\begin{array}{rrrrr}2 & 5 & 2 & 4 & 4 \\ -7 & 6 & 2 & 0 & 0\end{array}$

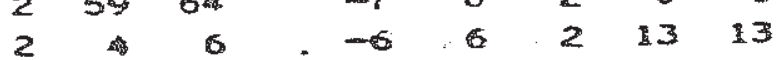

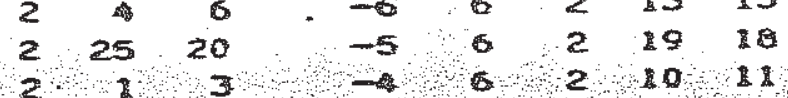




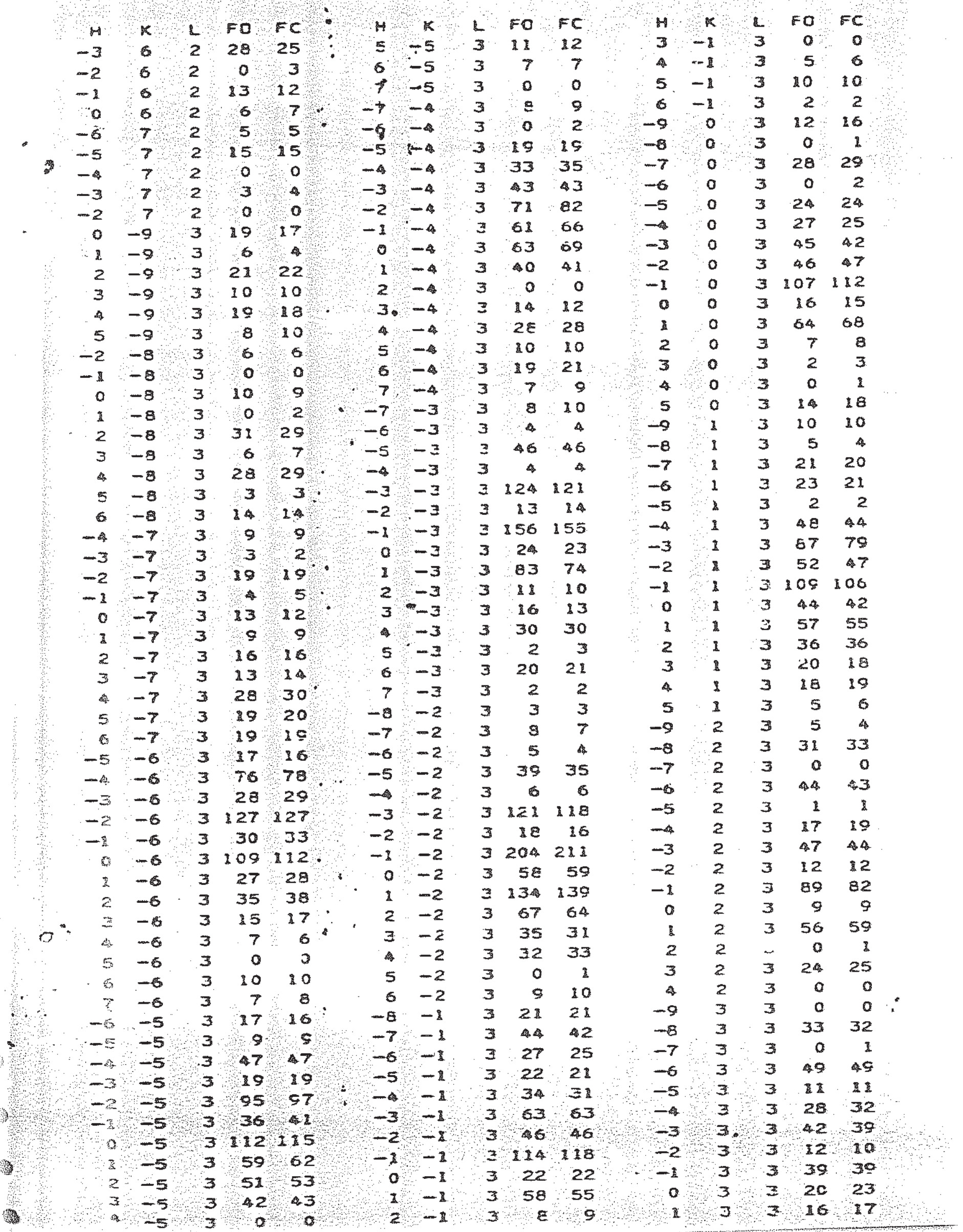




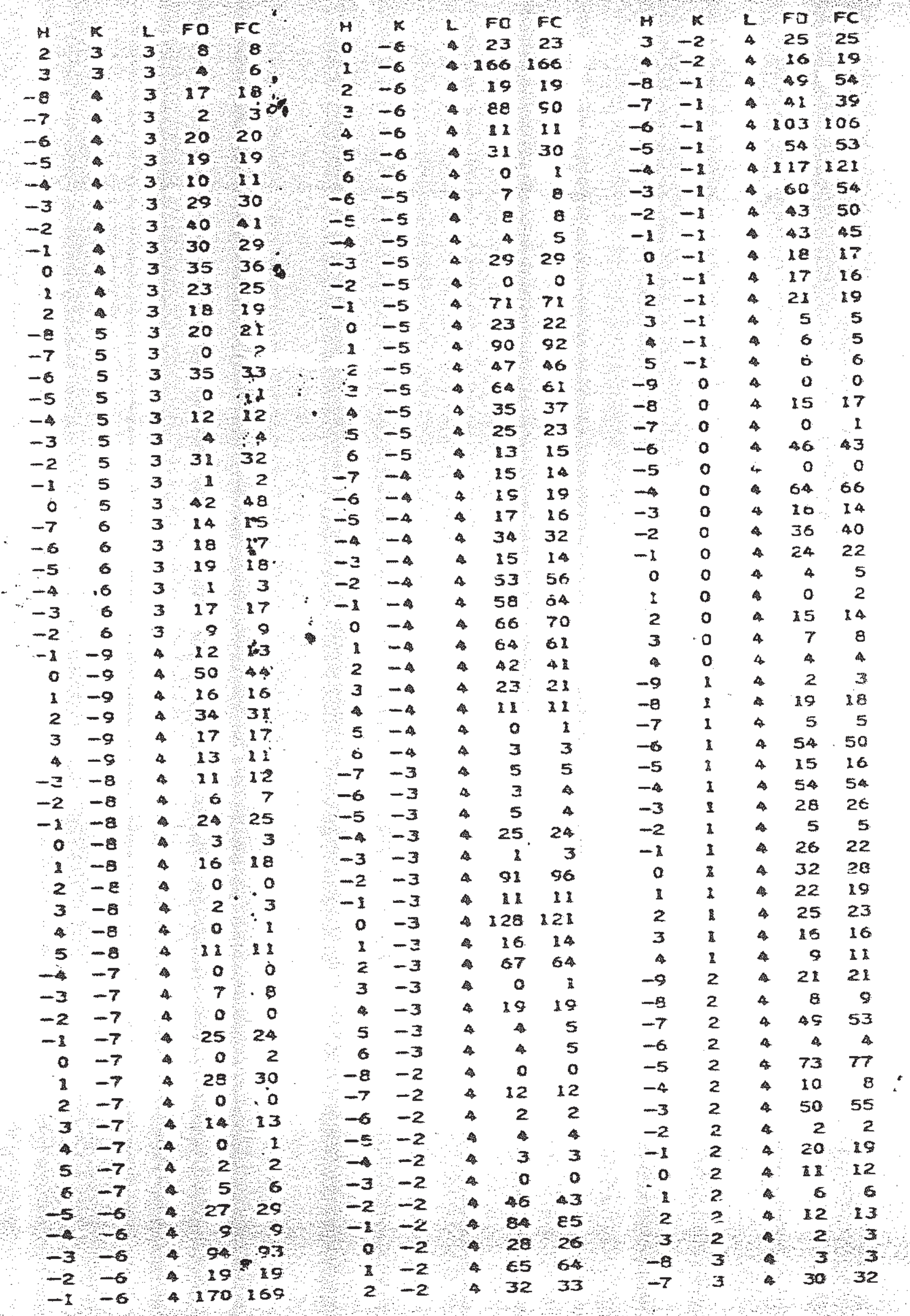




\begin{tabular}{|c|c|c|c|c|c|c|c|c|c|c|c|c|c|c|c|c|}
\hline$\sum_{i}$ & $\mathbb{N}$ & $x$ & 2 & $\mathrm{FO}$ & $F C$ & & $H$ & $x$ & $\mathrm{C}$ & $\mathrm{FO}$ & $\mathrm{FC}$ & 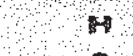 & 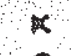 & $L$ & FO & $F C$ \\
\hline & -6 & 3 & 4 & 7 & 7 & & -1 & -5 & 5 & 16 & 17 & -8 & 0 & 5 & 5 & $\approx$ \\
\hline & -5 & 3 & 4 & 52 & 56 & & 0 & -5 & 5 & $\approx 7$ & 24 & -7 & 0 & 5 & 2 & 1 \\
\hline 5 & $-\infty$ & 3 & 4 & 0 & 0 & & 1 & -5 & 5 & 11 & I & -6 & 0 & 5 & 7 & 7 \\
\hline & -3 & 3 & 4 & 52 & 53 & & 2 & -5 & 5 & 42 & 36 & -5 & 0 & 5 & 24 & 21 \\
\hline & -2 & 3 & 4 & 15 & 184 & & $z$ & $-\leq$ & 5 & 24 & 22 & -4 & 0 & 5 & 2 & 4 \\
\hline 8 & -2 & 3 & 4 & 26 & 23 & & $\Leftrightarrow$ & -5 & 5 & 29 & 28 & -3 & $c$ & 5 & $\$ 6$ & 48 \\
\hline & 0 & 3 & $\Delta$ & 14 & 15 & & 5 & -5 & 5 & 19 & 21 & -2 & 0 & 5 & 12 & 12 \\
\hline & 1 & 3 & 4 & 5 & 3 & $\bullet$ & -7 & -4 & 5 & 111 & 11 & -1 & 0 & 5 & $\triangle 4$ & 6 \\
\hline & 2 & 3 & $\Delta$ & 6 & $7:$ & & -6 & -4 & 5 & 31 & 30 & 0 & 0 & 5 & 18 & 7 \\
\hline & -8 & $\Rightarrow$ & 8 & 4 & .5 & & -5 & -4 & 5 & 25 & 28 & 1 & 0 & 5 & 27 & 2 \\
\hline & -7 & 8 & 4 & 19 & 20 & & -4 & -4 & 5 & $\Delta 7$ & 51 & 2 & 0 & 5 & 6 & \\
\hline & $-c$ & 4 & 4 & 5 & 6 & 1 & -3 & -4 & 5 & 32 & 34 & 3 & 0 & 5 & 7 & \\
\hline & $\begin{array}{l}-5 \\
-5\end{array}$ & 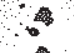 & 4 & $\Delta$ & 5 & : & $-\bar{z}$ & -4 & 5 & $2 E$ & 36 & -9 & 1 & 5 & 9 & \\
\hline & & 5 & 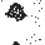 & 6 & 3 & 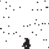 & -1 & -4 & 5 & 38 & $\$ 0$ & -7 & $\mathbf{I}$ & 5 & 10 & \\
\hline & -6 & 5 & 4 & 9 & 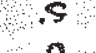 & 4 & 0 & -4 & 5 & 3 & $\begin{array}{l}1 \\
70\end{array}$ & $\begin{array}{l}-6 \\
-5\end{array}$ & 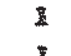 & 5 & 12 & II \\
\hline & -5 & 5 & 4 & $\begin{array}{r}0 \\
24\end{array}$ & 26 & & $\frac{1}{2}$ & $-\infty$ & $\begin{array}{l}5 \\
5\end{array}$ & $\begin{array}{l}45 \\
84\end{array}$ & $\begin{array}{l}35 \\
13\end{array}$ & $\begin{array}{l}-5 \\
-4\end{array}$ & 1 & $\begin{array}{l}5 \\
5\end{array}$ & 14 & $\begin{array}{l}40 \\
15\end{array}$ \\
\hline & -4 & 5 & 4 & 0 & 2 & & $z$ & -4 & 5 & 23 & 22 & -3 & 1 & 5 & 56 & 52 \\
\hline & -3 & 5 & 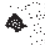 & 23 & 26 & & 4 & -4 & 5 & 6 & 6 & -2 & 1 & 5 & 16 & is \\
\hline & -2 & 5 & 4 & 5 & 16 & & 5 & $-\infty$ & 5 & 6 & 6 & -2 & 2 & 5 & 32 & 27 \\
\hline & $\begin{array}{r}-1 \\
0\end{array}$ & -9 & 5 & 34 & 38 & & -7 & -3 & 5 & $\varepsilon$ & 9 & 0 & 1 & 5 & 28 & 9 \\
\hline & 2 & -9 & 5 & 8 & 7 & & -6 & -3 & 5 & 15 & 16 & 1 & 1 & 5 & 2 & \\
\hline (7) & 2 & -9 & 5 & 56 & 49 & & -5 & -3 & 5 & 30 & 28 & 2 & 8 & 5 & 6 & 5 \\
\hline & $\geq$ & $\begin{array}{l}-9 \\
-9\end{array}$ & $\begin{array}{l}5 \\
5\end{array}$ & 9 & 93 & 3 & -4 & -3 & 5 & Ye & 19 & -8 & 2 & 5 & 12 & 23 \\
\hline & -3 & -2 & 5 & $\begin{array}{r}33 \\
8\end{array}$ & 39 & . & -3 & -3 & 5 & 26 & 26 & -7 & 2 & 5 & 10 & $\mathrm{~B}$ \\
\hline & -2 & -8 & 5 & 8 & 3 & $\bullet$ & -2 & -3 & $\begin{array}{l}5 \\
5\end{array}$ & 15 & 12 & $\begin{array}{l}-6 \\
-5\end{array}$ & $\begin{array}{l}2 \\
2\end{array}$ & $\begin{array}{l}5 \\
5\end{array}$ & $\begin{array}{r}1 \\
9\end{array}$ & 43 \\
\hline & -1 & -8 & 5 & II & II & & 0 & -3 & 3 & $\begin{array}{r}17 \\
9\end{array}$ & $\begin{array}{r}14 \\
8\end{array}$ & $\begin{array}{l}-5 \\
-4\end{array}$ & 2 & 5 & $\begin{array}{r}9 \\
60\end{array}$ & $\begin{array}{r}9 \\
63\end{array}$ \\
\hline & 0 & -8 & 5 & 25 & 23 & & 1 & -3 & 5 & $\begin{array}{r}9 \\
41\end{array}$ & $\begin{array}{r}8 \\
3 E\end{array}$ & -3 & 2. & 5 & 0 & $\begin{array}{r}0.3 \\
0\end{array}$ \\
\hline & 1 & -8 & 5 & 7 & 7 & & 2 & -3 & 5 & 4 & $\Delta$ & -2 & $z^{2}$ & 5 & $\$ 6$ & 4 \\
\hline & 2 & -8 & 5 & 24 & 24 & & 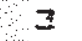 & -3 & 5 & 27 & 25 & -1 & 2 & 5 & 3 & 3 \\
\hline & 3 & -8 & 5 & 0 & 2 & & 4 & -3 & 5 & $a$ & 0 & 0 & 2 & 5 & 22 & 28 \\
\hline & 4 & -8 & 5 & 7 & 8 & & 5 & -3 & 5 & 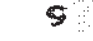 & 10 & 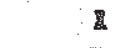 & 2 & 5 & $a$ & 0 \\
\hline & -4 & -7 & 5 & 0 & 0 & & -8 & -2 & 5 & 18 & 10 & -7 & 3 & 5 & 5 & 7 \\
\hline . & $\begin{array}{l}-3 \\
-2\end{array}$ & -7 & 5 & 14 & 12 & & -7 & -2 & 5 & 2 & 3 & -8 & 3 & 5 & 22 & 12 \\
\hline & $\begin{array}{l}-2 \\
-1\end{array}$ & $\begin{array}{l}-7 \\
-7\end{array}$ & $\begin{array}{l}5 \\
5\end{array}$ & 2 & 2 & & -6 & -2 & 5 & 16 & 15 & -5 & 3 & 5 & 7 & b \\
\hline & 0 & & $\begin{array}{l}5 \\
5\end{array}$ & 14 & 13 & & -5 & $-z$ & 5 & 0 & 0 & -4 & 3 & 5 & 26 & 29 \\
\hline & I & -7 & 5 & 22 & 12 & & -4 & -2 & 5 & 12 & 10 & -3 & 3 & $\Xi$ & 0 & 8 \\
\hline & z & -7 & 5 & 18 & $\begin{array}{r}5 \\
18\end{array}$ & & -3 & -2 & 5 & $\$$ & 9 & -2 & 3 & 5 & $\begin{array}{r}29 \\
8\end{array}$ & 82 \\
\hline & 3 & -7 & 5 & 7 & $\begin{array}{r}15 \\
5\end{array}$ & & $-\frac{\pi}{2}$ & $-z$ & 5 & $E$ & 9 & $\begin{array}{r}-1 \\
0\end{array}$ & $\begin{array}{l}3 \\
3\end{array}$ & $\begin{array}{l}5 \\
5\end{array}$ & $\begin{array}{r}8 \\
18\end{array}$ & 89 \\
\hline 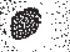 & $B$ & -7 & 5 & 18 & 18 & & -2 & $\begin{array}{l}-2 \\
-2\end{array}$ & 5 & 6 & 6 & -6 & 4 & 5 & 8 & 19 \\
\hline & 5 & -7 & 5 & 5 & 6 & & 1 & $-z$ & 5 & 75 & IH & -5 & 4 & 5 & 13 & 11 \\
\hline & - 5 & -6 & 5 & $\triangle$ & 4 & & 2 & -2 & 5 & 8 & 7 & $\rightarrow$ & 4 & 5 & $\mathbb{1} \mathbb{1}$ & 13 \\
\hline & $\rightarrow$ & -6 & 5 & 0 & 0 & & 3 & -2 & 5 & 17 & 87 & -3 & 4 & 5 & 5 & 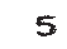 \\
\hline & -3 & -6 & 5 & 4 & 4 & & $\Delta$ & -2 & 5 & 18 & 12 & -2 & 4 & 5 & 12 & 13 \\
\hline & -2 & -6 & 5 & 49 & 44 & & -8 & -1 & 5 & 16 & I 7 & -2 & $-\varepsilon$ & 6 & 5 & 7 \\
\hline & -1 & -6 & 5 & 0 & 0 & & -7 & -1 & 5 & $5 \varepsilon$ & $6 I$ & -1 & -8 & $\sigma$ & 87 & 16 \\
\hline & $\therefore$ & -6 & 5 & 106 & 104 & & -6 & -1 & 5 & 35 & 39 & 0 & -8 & 6 & 83 & 10 \\
\hline & 2 & -6 & 5 & 0 & I & & -5 & -1 & 5 & 839 & 137 & I & -8 & 6 & 0 & 0 \\
\hline & $\begin{array}{l}2 \\
3\end{array}$ & -6 & 5 & 109 & $\$ 09$ & & $-B$ & -1 & 5 & 51 & 54 & 2 & -7 & 6 & 3 & $\varepsilon$ \\
\hline & 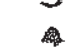 & $\begin{array}{l}-6 \\
-6\end{array}$ & 5 & 4 & 3 & & -3 & -2 & 5 & 155 & 169 & 3 & $-\varepsilon$ & 6 & 7 & E \\
\hline 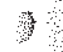 & 5 & $\begin{array}{l}-6 \\
-6\end{array}$ & $\begin{array}{l}5 \\
5\end{array}$ & $\ll 5$ & 58 & & -2 & -5 & 5 & $\begin{array}{l}45 \\
102\end{array}$ & 47 & $\begin{array}{l}-4 \\
-3\end{array}$ & $\begin{array}{l}-7 \\
-7\end{array}$ & 6 & $\begin{array}{r}12 \\
5\end{array}$ & $\begin{array}{r}10 \\
5\end{array}$ \\
\hline & -8 & -5 & 5 & $\frac{2}{4}$ & 3 & & $\begin{array}{r}-1 \\
0\end{array}$ & $a_{-1}^{-1}$ & $\begin{array}{l}5 \\
5\end{array}$ & 32 & 30 & -2 & -7 & 6 & 9 & 27 \\
\hline & -5 & -5 & 5 & 17 & IE, & & 2 & -1 & 5 & 66 & 41 & -1 & -7 & . & $\Leftrightarrow$ & 5 \\
\hline & -4 & -5 & 5 & 5 & 6 & & 2 & -1 & 5 & 21 & $\mathrm{IE}$ & 0 & -7 & 6 & 22 & 19 \\
\hline & -3 & -5 & 5 & 18 & 20 & & 3 & -3 & 5 & 12 & 9 & I & -7 & 6 & 3 & 4 \\
\hline & -2 & -5 & 5 & 10 & 9 & & 4 & -2 & 5 & 00 & 0 & 2 & -7 & 6 & 1 & 10 \\
\hline
\end{tabular}




\begin{tabular}{|c|c|c|c|c|c|c|c|c|c|c|c|c|c|c|c|}
\hline 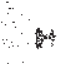 & $k$ & $L$ & $F$ & FC & & $M$ & $x$ & $L$ & $\mathrm{FO}$ & FC & $H$ & k & 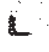 & FO & $F C$ \\
\hline-5 & -7 & 6 & 3 & 5 & & 2 & -4 & 4 & 16 & 16 & -1 & -1 & 6 & 24 & 23 \\
\hline $\begin{array}{l}-5 \\
-3\end{array}$ & $-\infty$ & 6 & 14 & 14 & & 3 & -4 & 6 & 10 & 8 & 0 & -1 & 6 & 78 & eo \\
\hline-4 & -6 & 6 & $?$ & 7 & & 4 & -4 & 6 & $e$ & 9 & 1 & -1 & 6 & 15 & 14 \\
\hline-2 & -6 & 6 & 27 & 28 & 0 & -7 & -3 & 6 & 9 & 10 & 2 & -1 & 6 & 38 & 8 \\
\hline $\begin{array}{l}-2 \\
-1\end{array}$ & -6 & 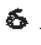 & 9 & 8 & 6 & -6 & -3 & 6 & 0 & 0 & -8 & 0 & 6 & 2 & \\
\hline $\begin{array}{r}-2 \\
0\end{array}$ & -6 & 6 & 15 & 14 & 3 & -5 & -3 & 6 & 20 & 22 & -7 & 0 & 6 & 14 & 14 \\
\hline s & -6 & 6 & 8 & 6 & 8 & -4 & -3 & 6 & 25 & 25 & -6 & 0 & 6 & 9 & \\
\hline 2 & $\begin{array}{l}-8 \\
-6\end{array}$ & 6 & 23 & 20 & & -3 & -3 & 6 & 26 & 25 & -5 & 0 & 6 & 16 & \\
\hline 3 & $\begin{array}{l}-6 \\
-6\end{array}$ & 6 & 3 & 4 & & -2 & -3 & 6 & 16 & 15 & -4 & 0 & 6 & 4 & \\
\hline sis & -6 & 6 & $\begin{array}{r}32 \\
2\end{array}$ & 35 & & -1 & -3 & 6 & 17 & $\begin{array}{r}15 \\
6\end{array}$ & $\begin{array}{l}-3 \\
-2\end{array}$ & 0 & 6 & a & \\
\hline - & -5 & 6 & 11 & $\begin{array}{r}2 \\
11\end{array}$ & & 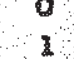 & $\begin{array}{l}-3 \\
-3\end{array}$ & 6 & $\begin{array}{l}5 \\
3\end{array}$ & 3 & $\begin{array}{l}-2 \\
-1\end{array}$ & $\begin{array}{l}0 \\
0\end{array}$ & $\begin{array}{l}6 \\
6\end{array}$ & $\begin{array}{r}12 \\
7\end{array}$ & o. \\
\hline$-E$ & -5 & 6 & 12 & 11 & & 2 & -3 & 6 & 2 & 2 & 0 & 0 & 6 & 87 & 17 \\
\hline $\begin{array}{l}-2 \\
-3\end{array}$ & -5 & 6 & 25 & 17 & & -7 & -2 & 6 & 0 & 1 & 1 & 0 & 5 & 18 & 8 \\
\hline-2 & -5 & 6 & 13 & 14 & & -6 & -2 & 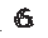 & 9 & 7 & -7 & I & 6 & 14 & 12 \\
\hline $\begin{array}{l}-2 \\
-8\end{array}$ & -5 & 6 & 12 & 22 & & -5 & -2 & 6 & 7 & 6 & -6 & 1 & 6 & 13 & 11 \\
\hline $\begin{array}{r}-\frac{1}{2} \\
5\end{array}$ & -5 & 6 & 5 & 2 & & -4 & -2 & 6 & e & 9 & -5 & 1 & 6 & 16 & \\
\hline 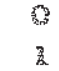 & -5 & 6 & 22 & 22 & & -3 & -2 & 6 & 14 & 10 & -4 & 1 & 6 & 6 & \\
\hline 3 & -5 & 6 & 8 & 7 & & -2 & -2 & 6 & 0 & 0 & -3 & 1 & 6 & 15 & \\
\hline 3 & -5 & 6 & 12 & 10 & & -3 & -2 & 6 & 15 & 26 & -2 & 1 & 6 & $1 \varepsilon$ & \\
\hline 3 & -5 & 6 & 8 & 5 & & 0 & -2 & 6 & $z$ & 2 & -1 & 1 & 0 & 11 & \\
\hline-7 & $\begin{array}{l}-5 \\
-4\end{array}$ & 6 & 3 & 3 & & 1 & -2 & 6 & 84 & 24 & c & 1 & 6 & 13 & 11 \\
\hline-6 & - & 6 & 8 & 21 & & 2 & -2 & 6 & 0 & 1 & -7 & 2 & 6 & $\begin{array}{l}3 \\
0\end{array}$ & \\
\hline-5 & -4 & 6 & 38 & 34 & & 3 & -2 & 6 & 4 & 4 & $\begin{array}{l}-8 \\
-5\end{array}$ & 2 & 6 & 11 & \\
\hline-4 & -2 & 6 & 88 & 19 & & $\begin{array}{l}-8 \\
-7\end{array}$ & -1 & $\begin{array}{l}6 \\
6\end{array}$ & $\begin{array}{l}0 \\
6\end{array}$ & 6 & -4 & 2 & 6 & 3 & \\
\hline-3 & -4 & 6 & 53 & $5 \%$ & & -6 & -2 & 6 & 27 & 26 & -3 & 2 & 6 & 24 & \\
\hline-2 & -4 & 6 & 18 & 23 & & -5 & -8 & 6 & 15 & 10 & -2 & 2 & 6 & 1 & \\
\hline-3 & $\rightarrow$ & 6 & 52 & 54 & & -4 & $-\mathbf{2}$ & 6 & $8 \geq$ & 2. & -1 & 2 & 6 & 19 & \\
\hline 0 & - & 6 & $\approx 1$ & 21 & & -3 & -1 & 6 & 27 & 26 & -5 & 3 & 6 & 0 & \\
\hline & -4 & 6 & 30 & 26 & & -2 & -1 & $\epsilon$ & 107 & 108 & -4 & 3 & 6 & 9 & \\
\hline
\end{tabular}

